\title{
La sismicità nella zona di Nocera Umbra nel periodo maggio-luglio 1979.
}

Seismicity in the Nocera Umbra area during May-July 1979 period.

\author{
Biagi P.F.* - Console R.** - Della Monica G. - Iuzzolini P.* \\ Lazzi M. - Piccone italiano A. - Piro S.
}

Ricevuto il 10 Settembre, 1979

\section{ABstract}

We are going to describe the seismic crisis which has interested Nocera Umbra area (Perugia) from the middle of May to the beginning of August 1979, which has been recorded by a seismic station, installed in the town.

Various events have had magnitude ranging from 1.3 to 2.9 . The registrata da una stazione sismica installata nel paese.

approximately.

Data' elaboration has allowed to point out that the hypocentre' depth increases, considerably, from Est to West.

At last, it represents a possible correlation between observed distribution and geological structures of the interested area.

${ }^{\star}{ }^{*}$ Istituto di Geologia e Paleontologia, Università degli studi Roma, Piazzale Aldo Moro 5.

${ }^{* *}$ Istituto Nazionale di Geofisica, Osservatorio Centrale di Monte Porzio Catone. 


\section{RIASSUNTO}

Si descrive la crisi sismica che ha interessato la zona di Nocera Umbra (Perugia) dalla metà di maggio ai primi di agosto 1979, quale è stata registrata da una stazione sismica installata nel paese.

I vari eventi hanno avuto magnitudo compresa fra 1,3 e 2,9. La profondità degli ipocentri è risultata assai piccola, variabile fra $0,5 \mathrm{Km} e$ $5 \mathrm{Km}$ circa.

L'elaborazione dei dati ha permesso di rilevare che la profondità ipocentrale aumenta, in maniera significativa, da Est ad Ovest.

$\mathrm{E}^{\prime}$ infine presentata una possibile correlazione fra la distribuzione osservata e le strutture geologiche esistenti nella zona. 


\section{INTRODUZIONE}

Dalla metà del maggio 1979 l'area compresa fra Gualdo Tadino, Foligno, Spoleto, Cascia, Norcia e Camerino è stata interessata da una consistente attività sismica.

Nella Tabella I sono riportati i dati riguardanti le scosse principali verificatesi in detta area fino alla fine di luglio, quali sono stati ricavati dalle registrazioni ottenute in alcune stazioni della rete sismica nazionale. Gli epicentri relativi sono riportati nella carta di fig. 1.

Le scosse del 18, 19 e 21 maggio sono state avvertite in particolar modo nella zona di Nocera Umbra, cioè in quella parte Nord-orientale dell'Umbria situata a metà dell'arco che forma l'Appennino Umbro-Marchigiano, dove i rilievi cambiano decisamente il proprio allineamento geografico. In questa area tali eventi hanno segnato l'inizio di una frequente attività sismica che ha messo spesso in allarme la popolazione.

L'attività suddetta si è presentata subito estremamente localizzata e caratterizzata da terremoti di piccola magnitudo, sì che le registrazioni ottenute nelle stazioni sismiche dell'I.N.G. più vicine non erano in grado di definirne gli aspetti caratteristici. Per questo motivo, a partire dai primi giorni del mese di giugno si è provveduto ad installare una stazione sismica a Nocera Umbra, nella parte alta del paese presso la sede del Comune.

1. La strumentazione impiegata è costituita da una terna di sismografi a breve periodo con amplificazione elettronica e registrazione su carta termosensibile. Il gruppo dei registratori è montato su autofurgone, rendendo possibile il trasferimento della stazione in tempi relativamente brevi.

I sensori sono di tipo S-13, ad un secondo di periodo proprio. Gli amplificatori hanno frequenze di taglio a $0.1 \mathrm{~Hz}$ e $5 \mathrm{~Hz}$ e consentono un guadagno variabile fra limiti assai ampi con passi di $6 \mathrm{db}$. I registratori hanno una velocità di scorrimento di $60 \mathrm{~mm} / \mathrm{min}$ per una durata di $24 \mathrm{~h}$. Inoltre la stazione è dotata di un orologio marcatempo e di un gruppo alimentatore. 
T A B E L L A 1

\begin{tabular}{|c|c|c|c|c|c|c|c|}
\hline Data & & & $H$ & $\varphi$ & $\lambda$ & $h(\mathrm{Km})$ & $M$ \\
\hline $18 / 4$ & 22 & 14 & $0.7 \pm 0.53$ & $43.106 \pm 0.032$ & $12.738 \pm 0.055$ & $5 \pm 6.6$ & 2.8 \\
\hline $19 / 5$ & 7 & 19 & $4.4 \pm 1.26$ & $42.925 \pm 0.080$ & $13.093 \pm 0.120$ & $39 \pm 15.0$ & 3.3 \\
\hline $19 / 5$ & 8 & 8 & $37.5+1.26$ & $43.084 \pm 0.016$ & $12.802 \pm 0.058$ & $45 \pm 8.0$ & 3.0 \\
\hline $21 / 5$ & 12 & 26 & $13.5 \pm 0.46$ & $43.120 \pm 0.029$ & $12.866 \pm 0.046$ & $11+4.3$ & 3.3 \\
\hline $21 / 5$ & 12 & 36 & $40.8 \pm 0.33$ & $43.085 \pm 0.023$ & $12.804 \pm 0.035$ & $13 \pm 3.4$ & 3.5 \\
\hline $21 / 5$ & 14 & 34 & $16.9 \pm 0.35$ & $43.050 \pm 0.030$ & $12.962+0.044$ & $33 \pm 4.8$ & 3.6 \\
\hline $21 / 5$ & 17 & 32 & $10.0 \pm 0.46$ & $43.084 \pm 0.027$ & $12.851+0.043$ & $13 \pm 3.9$ & 3.3 \\
\hline $22 / 5$ & 0 & 28 & $55.1+0.50$ & $43.131 \pm 0.030$ & $12.840 \pm 0.055$ & $10 \pm 6.0$ & 3.1 \\
\hline $30 / 5$ & 1 & 11 & $36.9+0.85$ & $43.061 \pm 0.049$ & $12.756 \pm 0.087$ & $11 \pm 8.8$ & 3.0 \\
\hline $30 / 5$ & 12 & 22 & $40.4 \pm 0.97$ & $43.035 \pm 0.046$ & $12.861 \pm 0.100$ & $5 \pm 12.1$ & 3.2 \\
\hline $1 / 6$ & 15 & 50 & $39.3+2.38$ & $43.048 \pm 0.035$ & $12.934 \pm 0.070$ & $35 \pm 20.5$ & 3.7 \\
\hline $16 / 6$ & 19 & 40 & $0.3 \pm 0.48$ & $43.145 \pm 0.020$ & $12.944 \pm 0.030$ & $9 \pm 3.5$ & 3.9 \\
\hline $17 / 6$ & 4 & 59 & $36.1+0.64$ & $43.153 \pm 0.023$ & $12.881+0.043$ & $1 \pm 4.8$ & 3.8 \\
\hline $19 / 6$ & 22 & 58 & $27.5 \pm 0.82$ & $42.887 \pm 0.023$ & $13.041 \pm 0.015$ & $47 \pm 6.6$ & \\
\hline $19 / 6$ & 23 & 5 & $4.8 \pm 1.40$ & $42.932 \pm 0.016$ & $12.998 \pm 0.010$ & $27 \pm 4.5$ & \\
\hline $20 / 6$ & 0 & 45 & $18.2 \pm 1.40$ & $42.940 \pm 0.054$ & $12.968 \pm 0.030$ & $20 \pm 20.0$ & \\
\hline $26 / 6$ & 19 & 3 & $54.8+0.72$ & $43.114 \pm 0.020$ & $12.817 \pm 0.015$ & $25 \pm 10.2$ & 3.0 \\
\hline $27 / 6$ & 8 & 27 & $26.2 \pm 0.04$ & $43.116 \pm 0.001$ & $12.809 \pm 0.001$ & $39 \pm 0.3$ & \\
\hline $28 / 6$ & 5 & 43 & $10.7 \pm 0.77$ & $43.138 \pm 0.046$ & $12.858 \pm 0.037$ & $2 \pm 7.8$ & 2.9 \\
\hline $29 / 6$ & 18 & 37 & $1.7 \pm 0.72$ & $43.199 \pm 0.070$ & $12.843 \pm 0.038$ & $15 \pm 9.8$ & \\
\hline $4 / 7$ & 14 & 7 & $55.9 \pm 2.75$ & $43.394+0.127$ & $12.886 \pm 0.085$ & $39+15.2$ & 2.5 \\
\hline $6 / 7$ & 22 & 17 & $8.3 \pm 0.71$ & $42.933 \pm 0.024$ & $12.997+0.041$ & $21 \pm 6.4$ & 3.5 \\
\hline $22 / 7$ & 22 & 21 & $28.6 \pm 1.06$ & $42.461+0.051$ & $12.798 \pm 0.025$ & $0 \pm 0.0$ & 2.2 \\
\hline
\end{tabular}


T A B E L L A 2

\begin{tabular}{|c|c|c|c|c|c|}
\hline Data & $H$ & $\varphi$ & $\lambda$ & $h(\mathrm{Km})$ & $M$ \\
\hline $13 / 6$ & $1410^{\prime}$ & $43^{\circ} 07^{\prime} 28^{\prime \prime}$ & $12^{\circ} 46^{\prime} 26^{\prime \prime}$ & 2,8 & 2.1 \\
\hline $13 / 6$ & $2353^{\prime}$ & $43^{\circ} 05^{\prime} 34^{\prime \prime}$ & $12^{\circ} 49^{\prime} 22^{\prime \prime}$ & 1,4 & 1.5 \\
\hline $14 / 6$ & $1543^{\prime}$ & $43 \circ 05^{\prime} 11^{\prime \prime}$ & $12^{\circ} 50^{\prime} 10^{\prime \prime}$ & 1,9 & 1.3 \\
\hline $15 / 6$ & $1225^{\prime}$ & $43 \circ 07^{\prime} 31^{\prime \prime}$ & $1246^{\prime} 38^{\prime \prime}$ & 3,6 & 2.0 \\
\hline $15 / 6$ & $1357^{\prime}$ & $43^{\circ} 08^{\prime} 33^{\prime \prime}$ & $12^{\circ} 47^{\prime} 11^{\prime \prime}$ & 5,1 & 2.0 \\
\hline $15 / 6$ & $1932^{\prime}$ & $43^{\circ} 07^{\prime} 24^{\prime \prime}$ & $12^{\circ} 47^{\prime} 13^{\prime \prime}$ & 3,9 & 2.1 \\
\hline $16 / 6$ & $1036^{\prime}$ & $43^{\circ} 05^{\prime} 33^{\prime \prime}$ & $12^{\circ} 46^{\prime} 01^{\prime \prime}$ & 4,9 & 1.9 \\
\hline $16 / 6$ & $1541^{\prime}$ & $43^{\circ} 11^{\prime} 24^{\prime \prime}$ & $12^{\circ} 51^{\prime} 38^{\prime \prime}$ & 2,3 & 1.5 \\
\hline $16 / 6$ & $1628^{\prime}$ & $43^{\circ} 07^{\prime} 42^{\prime \prime}$ & $12^{\circ} 47^{\prime} 16^{\prime \prime}$ & 3,6 & 2.2 \\
\hline $22 / 6$ & $2338^{\prime}$ & $43^{\circ} 06^{\prime} 24^{\prime \prime}$ & $12^{\circ} 48^{\prime} 54^{\prime \prime}$ & 0,4 & 1.7 \\
\hline $23 / 6$ & $0746^{\prime}$ & $43^{\circ} 05^{\prime} 45^{\prime \prime}$ & $12^{\circ} 46^{\prime} 32^{\prime \prime}$ & 3,0 & 2.6 \\
\hline $23 / 6$ & 09 12' & $43 \circ 04^{\prime} 35^{\prime \prime}$ & $12^{\circ} 45^{\prime} 32^{\prime \prime}$ & 8,0 & 2.8 \\
\hline $23 / 6$ & $1240^{\prime}$ & $43^{\circ} 05^{\prime} 02^{\prime \prime}$ & $12^{\circ} 46^{\prime} 19^{\prime \prime}$ & 2,2 & 2.6 \\
\hline $23 / 6$ & $1452^{\prime}$ & $43^{\circ} 07^{\prime} 05^{\prime \prime}$ & $12^{\circ} 46^{\prime} 23^{\prime \prime}$ & 3,4 & 1.9 \\
\hline $24 / 6$ & $1156^{\prime}$ & $43^{\circ} 06^{\prime} 41^{\prime \prime}$ & $12^{\circ} 46^{\prime} 00^{\prime \prime}$ & 4,5 & 2.1 \\
\hline $24 / 6$ & 12 09' & $43^{\circ} 05^{\prime} 30^{\prime \prime}$ & $12^{\circ} 46^{\prime} 26^{\prime \prime}$ & 1,6 & 2.8 \\
\hline $24 / 6$ & $1312^{\prime}$ & $43^{\circ} 06^{\prime} 45^{\prime \prime}$ & $12^{\circ} 48^{\prime} 44^{\prime \prime}$ & 3,0 & 2.4 \\
\hline $24 / 6$ & $1341^{\prime}$ & $43^{\circ} 06^{\prime} 41^{\prime \prime}$ & $12^{\circ} 46^{\prime} 24^{\prime \prime}$ & 3,2 & 2.6 \\
\hline $24 / 6$ & $2250^{\prime}$ & $43^{\circ} 06^{\prime} 48^{\prime \prime}$ & $12^{\circ} 46^{\prime} 40^{\prime \prime}$ & 4,4 & 2.3 \\
\hline $25 / 6$ & $0211^{\prime}$ & $43^{\circ} 06^{\prime} 48^{\prime \prime}$ & $12^{\circ} 46^{\prime} 51^{\prime \prime}$ & 3,4 & 2.4 \\
\hline $25 / 6$ & $0758^{\prime}$ & $43^{\circ} 06^{\prime} 54^{\prime \prime}$ & $12^{\circ} 46^{\prime} 05^{\prime \prime}$ & 3,8 & 2.3 \\
\hline $25 / 6$ & $08 \quad 38^{\prime}$ & $43^{\circ} 06^{\prime} 39^{\prime \prime}$ & $12^{\circ} 46^{\prime} 06^{\prime \prime}$ & 4,6 & 2.8 \\
\hline $25 / 6$ & $1528^{\prime}$ & $43^{\circ} 07^{\prime} 30^{\prime \prime}$ & $12^{\circ} 45^{\prime} 45^{\prime \prime}$ & 4,0 & 1.9 \\
\hline $26 / 6$ & $16 \quad 05^{\prime}$ & $43^{\circ} 06^{\prime} 41^{\prime \prime}$ & $12^{\circ} 46^{\prime} 55^{\prime \prime}$ & 3,4 & 1.5 \\
\hline $26 / 6$ & $1904^{\prime}$ & $43^{\circ} 04^{\prime} 53^{\prime \prime}$ & $12^{\circ} 47^{\prime} 12^{\prime \prime}$ & 1,7 & 2.9 \\
\hline $26 / 6$ & $1921^{\prime}$ & $43^{\circ} 05^{\prime} 15^{\prime \prime}$ & $12^{\circ} 49^{\prime} 55^{\prime \prime}$ & 0,5 & 2.7 \\
\hline $26 / 6$ & $2124^{\prime}$ & $43^{\circ} 06^{\prime} 27^{\prime \prime}$ & $12^{\circ} 49^{\prime} 46^{\prime \prime}$ & 1,4 & 2.1 \\
\hline $27 / 6$ & $0423^{\prime}$ & $43^{\circ} 06^{\prime} 12^{\prime \prime}$ & $12^{\circ} 48^{\prime} 55^{\prime \prime}$ & 2,8 & 2.6 \\
\hline
\end{tabular}


(segue TAB. 2)

\begin{tabular}{|c|c|c|c|c|c|}
\hline Data & $H$ & $\varphi$ & $\lambda$ & $h(\mathrm{Km})$ & $M$ \\
\hline $27 / 6$ & $08 \quad 27^{\prime}$ & $43 \circ 07 ' 19 "$ & $12 \circ 45^{\prime} 09^{\prime \prime}$ & 1,8 & 2.9 \\
\hline $27 / 6$ & $08 \quad 29^{\prime}$ & $43^{\circ} 06^{\prime} 45^{\prime \prime}$ & $12^{\circ} 46^{\prime} 33^{\prime \prime}$ & 3,3 & 2.8 \\
\hline $27 / 6$ & $0831^{\prime}$ & $43^{\circ} 06^{\prime} 36^{\prime \prime}$ & $12^{\circ} 46^{\prime} 50^{\prime \prime}$ & 3,4 & 2.8 \\
\hline $27 / 6$ & $1016^{\prime}$ & $43^{\circ} 06^{\prime} 17^{\prime \prime}$ & $12^{\circ} 50^{\prime} 17^{\prime \prime}$ & 1,4 & 2.3 \\
\hline $27 / 6$ & $1143^{\prime}$ & $43006^{\prime} 42^{\prime \prime}$ & $12^{\circ} 47^{\prime} 00^{\prime \prime}$ & 4,2 & 2.5 \\
\hline $27 / 6$ & $1145^{\prime}$ & $43^{\circ} 06^{\prime} 34^{\prime \prime}$ & $12^{\circ} 46^{\prime} 57^{\prime \prime}$ & 4,2 & 2.8 \\
\hline $27 / 6$ & $2244^{\prime}$ & 43०07'30"' & $12 \circ 47^{\prime} 44^{\prime \prime}$ & 3,8 & 2.4 \\
\hline $5 / 7$ & $1940^{\prime}$ & $43^{\circ} 0800^{\prime \prime}$ & $12^{\circ} 47^{\prime} 09^{\prime \prime}$ & 3,2 & 2.5 \\
\hline $5 / 7$ & $2337^{\prime}$ & $43^{\circ} 07^{\prime} 28^{\prime \prime}$ & $12^{\circ} 46^{\prime} 48^{\prime \prime}$ & 3,7 & 2.1 \\
\hline $6 / 7$ & $2217^{\prime}$ & $43^{\circ} 05^{\prime} 42^{\prime \prime}$ & $12^{\circ} 46^{\prime} 44^{\prime \prime}$ & 2,2 & 2.7 \\
\hline $7 / 7$ & $0727^{\prime}$ & $43^{\circ} 07^{\prime} 03^{\prime \prime}$ & $12^{\circ} 47^{\prime} 09^{\prime \prime}$ & 5,2 & 2.0 \\
\hline $7 / 7$ & $2422^{\prime}$ & $43^{\circ} 07^{\prime} 44^{\prime \prime}$ & $12^{\circ} 46^{\prime} 59^{\prime \prime}$ & 3,5 & 2.3 \\
\hline $10 / 7$ & $0724^{\prime}$ & $43^{\circ} 07^{\prime} 28^{\prime \prime}$ & $12^{\circ} 47^{\prime} 11^{\prime \prime}$ & 3,4 & 2.2 \\
\hline $13 / 7$ & $0500^{\prime}$ & $43^{\circ} 06^{\prime} 04^{\prime \prime}$ & $12^{\circ} 46^{\prime} 45^{\prime \prime}$ & 4,4 & 2.0 \\
\hline $14 / 7$ & $2022^{\prime}$ & $43^{\circ} 06^{\prime} 19^{\prime \prime}$ & $12^{\circ} 50^{\prime} 21^{\prime \prime}$ & 1,2 & 2.0 \\
\hline $15 / 7$ & $1025^{\prime}$ & $43^{\circ} 06^{\prime} 19^{\prime \prime}$ & $12^{\circ} 47^{\prime} 07^{\prime \prime}$ & 3,8 & 2.2 \\
\hline $16 / 7$ & $2240^{\prime}$ & $43^{\circ} 06^{\prime} 42^{\prime \prime}$ & $12^{\circ} 47^{\prime} 41^{\prime \prime}$ & 35 & 2.5 \\
\hline $18 / 7$ & $0156^{\prime}$ & $43^{\circ} 06^{\prime} 42^{\prime \prime}$ & $12^{\circ} 47^{\prime} 00^{\prime \prime}$ & 3,5 & 2.2 \\
\hline $18 / 7$ & $1321^{\prime}$ & $43^{\circ} 07^{\prime} 03^{\prime \prime}$ & $12 \circ 50^{\prime} 49^{\prime \prime}$ & 1,3 & 1.7 \\
\hline $19 / 7$ & $0523^{\prime}$ & $43^{\circ} 06^{\prime} 43^{\prime \prime}$ & $12^{\circ} 48^{\prime} 50^{\prime \prime}$ & 2,0 & 1.7 \\
\hline $19 / 7$ & 0907 & $43^{\circ} 0537^{\prime \prime}$ & $12^{\circ} 47^{\prime} 08^{\prime \prime}$ & 3,8 & 2.2 \\
\hline $19 / 7$ & $1356^{\prime}$ & $43^{\circ} 08^{\prime} 27^{\prime \prime}$ & $12^{\circ} 46^{\prime} 19^{\prime \prime}$ & 5,0 & 1.8 \\
\hline $20 / 7$ & $0757^{\prime}$ & $43^{\circ} 05^{\prime} 37^{\prime \prime}$ & $12^{\circ} 46^{\prime} 57^{\prime \prime}$ & 3,0 & 2.3 \\
\hline $20 / 7$ & $1545^{\prime}$ & $43^{\circ} 05^{\prime} 57^{\prime \prime}$ & $12^{\circ} 46^{\prime} 59^{\prime \prime}$ & 2,5 & 2.3 \\
\hline $23 / 7$ & $0337^{\prime}$ & $43^{\circ} 07^{\prime} 48^{\prime \prime}$ & $12 \circ 46^{\prime} 45^{\prime \prime}$ & 3,8 & 2.4 \\
\hline $24 / 7$ & $044^{\prime}$ & $43^{\circ} 06^{\prime} 30^{\prime \prime}$ & $12^{\circ} 46^{\prime} 57^{\prime \prime}$ & 3,3 & 2.5 \\
\hline $24 / 7$ & $044^{\prime}$ & $43^{\circ} 06^{\prime} 11^{\prime \prime}$ & $12^{\circ} 49^{\prime} 04^{\prime \prime}$ & 0,7 & 2.2 \\
\hline $25 / 7$ & $0345^{\prime}$ & $43 \circ 07^{\prime} 41^{\prime \prime}$ & $12 \circ 48^{\prime} 19^{\prime \prime}$ & 3,4 & 2.3 \\
\hline $26 / 7$ & $2249^{\prime}$ & $43^{\circ} 06^{\prime} 21^{\prime \prime}$ & $12^{\circ} 47^{\prime} 38^{\prime \prime}$ & 1,7 & 1.9 \\
\hline
\end{tabular}


La curva di risposta dei sismografi ha un massimo intorno a $5 \mathrm{~Hz}$, in corrispondenza del quale si ha un'amplificazione complessiva di circa 2500 volte alla massima attenuazione. Le varie parti sono tarate in modo da poter considerare le curve di risposta delle tre componenti praticamente uguali fra loro.

I sismografi a componente orizzontale sono stati precedentemente tarati mediante un confronto diretto con una coppia a torsione "Wood Anderson ". Pertanto è stato possibile determinare la magnitudo degli eventi sismici mediante la lettura delle ampiezze massime delle registrazioni.

In coincidenza dei terremoti più forti il sismografo si è spesso saturato con conseguente danno alle pennine del registratore. Le registrazioni ottenute sono quindi incomplete. In ogni caso si è potuto seguire con buona accuratezza l'attività sismica in atto. Nella fig. 2 è riprodotto l'istogramma rappresentante la frequenza giornaliera dei terremoti con magnitudo non inferiore a 1.5 .

Come è evidenziato dall'istogramma la crisi si è andata estinguendo verso i primi di agosto.

L'esame dei sismogrammi ottenuti ha permesso di interpretare buona parte degli eventi sismici verificatisi. I dati così ricavati sono quelli della Tabella 2 . Il tempo origine tabulato è solo indicativo a causa della mancanza di un corretto segnale orario.

Le determinazioni ipocentrali sono state effettuate con il "metodo delle tre componenti". Date le brevissime distanze fra ipocentri e stazione (inferiori generalmente ai $10 \mathrm{Km}$ ), gli inevitabili errori commessi nella determinazione dell'azimut sono traducibili in un'incertezza dell'ordine del chilometro sugli epicentri.

Nella fig. 3 sono riportati su carta gli epicentri relativi. Come si può osservare, i vari terremoti, che hanno avuto magnitudo compreso fra 1.3 e 2.9 si sono verificati in una zona abbastanza ristretta distribuita in una fascia diretta Nord-Sud che lambisce la parte occidentale di Nocera Umbra. La profondità degli ipocentri è risultata assai piccola, variabile fra $0,5 \mathrm{Km}$ e $5 \mathrm{Km}$ circa. 
2. Nella zona di Nocera Umbra affiorano terreni distinguibili principalmente in due tipi: uno più recente di carattere fliscioide che costituisce la cosiddetta formazione Marnosa-Arenacea di età Miocenica e che affiora ad occidente; l'altro più antico, ad Est, formato dalla serie prevalentemente carbonatica di facies pelagiche, che prende il nome di serie Umbro-Marchigiana, di età dal Lias al Miocene Inferiore (fig. 3).

Tutte le formazioni presenti nella zona sono state interessate da una intensa tettonica compressiva che si manifesta con pieghe rovesciate, pieghe faglie e faglie inverse.

La serie Umbro-Marchigiana mette meglio in evidenza $\mathrm{i}$ movimenti subìti grazie alla possibilità di distinguervi nettamente un certo numero di formazioni, caratterizzate da litologie ben differenziate. Infatti ad Est di Nocera Umbra sono state riconosciute successioni di pieghe anticlinali molto inclinate, sviluppate con direzione Nord-Sud e con vergenza ad Est. I fianchi occidentali di queste pieghe risultano inclinati lievemente, con pendenza dell'ordine di circa $30^{\circ}$.

In più luoghi è possibile osservare (Carta geologica d'Italia $\mathrm{F}^{\circ}$ 123-Assisi) che $\mathrm{i}$ fianchi orientali di queste pieghe sono troncati da faglie inverse di cui non è valutabile il rigetto, che appare comunque di notevole entità.

La formazione Marnosa-Arenacea sembra formare invece ad Ovest di Nocera Umbra una blanda struttura sinclinale fino a lambire il fianco Nord-occidentale della struttura del Monte Subasio.

Lo spessore conosciuto della serie Umbro-Marchigiana è di 2,5-3 Km, ma è da tenere presente che spesso le strutture anticlinali ricostruibili sono formate da parti incomplete della serie. Più difficile è valutare gli spessori della formazione MarnosaArenacea che comunque dovrebbero essere superiori al $\mathrm{Km}$; $60 \mathrm{Km}$ più a Nord-Ovest (Carta geologica d'Italia $\mathrm{F}^{\circ} 115$-Città di Castello) gli spessori di questa formazione sono valutati a $3 \mathrm{Km}$.

3. Si è infine cercato di correlare gli elementi strutturali dei terreni con la crisi sismica studiata.

Dall'esame della fig. 3 è possibile rilevare che gli ipocentri 
più profondi sono raggruppati nella fascia posta più ad Ovest di Nocera Umbra, mentre gli ipocentri posti più ad Est tendono ad avere profondità sempre più piccole.

In base a questa osservazione ed in considerazione che le strutture geologiche hanno un andamento Nord-Sud, gli ipocentri sono stati proiettati su una sezione verticale orientata Est-Ovest (fig. 4a).

Su tale sezione si evidenzia nettamente che i punti distribuiti più in basso sono ben allineati e con un andamento che ricalca quello dei più importanti elementi strutturali osservabili in superficie, cioè i fianchi occidentali delle pieghe e le principali faglie inverse (profilo geologico, carta geologica d'Italia $\mathrm{F}^{\circ}$ 123-Assisi). Collegando idealmente gli ipocentri distribuiti lungo questo allineamento e prolungando verso l'alto questa linea si viene quasi ad intercettare la faglia inversa che affiora sul fianco orientale del Monte Pennino. Questa particolarità ha orientato l'interpretazione verso una corrispondenza fra tale distribuzione e la presenza di superfici di discontinuità tettonica.

In base a queste considerazioni nella sezione riportata nella fig. $4 \mathrm{~b}$ viene rappresentata, insieme agli ipocentri, una estrapolazione verso il basso degli elementi strutturali osservabili in superficie.

Dalla ricostruzione schematica di tale figura si vede che c'è anche una parte degli ipocentri localizzati lontano dalle superfici di discontinuità tettonica evidenziata. Ciò può significare che anche discontinuità stratigrafiche possono essere state sedi di movimenti, tanto più che queste discontinuità, già in passato, possono essere state interessate da piccole dislocazioni tettoniche.

In conclusione si può dedurre che i sismi sono stati provocati da un aumento locale delle tensioni orizzontali che hanno favorito movimenti lungo piani inclinati meno di $45^{\circ}$. La presenza di una compressione orizzontale concentrata in un volume abbastanza superficiale sembra essere solo una conseguenza di movimenti a componente verticale che sono tuttora presenti nell'Appennino centrale. 


\section{BIBLIOGRAFIA}

Aurelio V., Console R., Gasparini, C., 1973 - La magnitudo calcolata mediante sismografi a breve periodo. "Annali di Geofisica ", XXVI, 4, 693-703.

BIASINI A., 1966 - Elementi morfotettonici, tratti da un rilievo fotogeolo. gico, al margine dell'altopiano di Ovindoli (Abruzzo). "Geologia Romana ", V, 303-311.

Bosi C., 1975 - Osservazioni preliminari su faglie probabilmente attive nell'Appennino centrale. "Boll. Soc. Geol. It.", 94, 827-859.

I.N.G., 1979 - Bollettini sismici provvisori della Rete Sismica nazionale, Maggio-luglio 1979.

Nardi L. NARdi R., 1975 - Structural pattern of the Northen Apennines. "La Ricerca scientifica", C.N.R., pp. 203-257.

Servizio Geologico d'Italia, 1968 - Carta geologica d'Italia alla scala 1: 100.000, $\mathrm{F}^{\circ}$ 115-Città di Castello.

Servizio Geologico d'Italia, 1968 - Carta geologica d'Italia alla scala 1: $100.000, \mathrm{~F}^{\circ}$ 123-Assisi. 


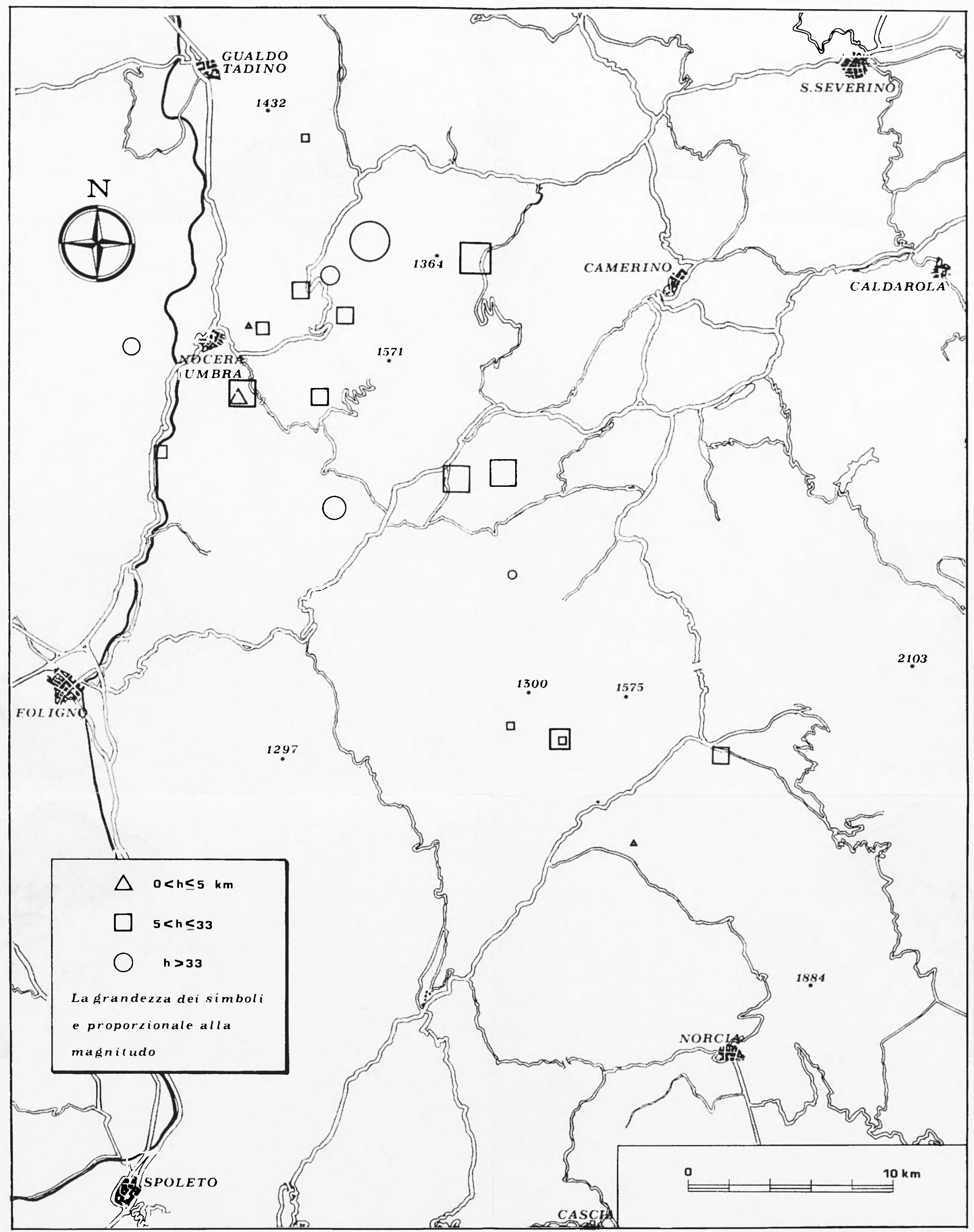

Fig. 1 - Rappresentazione degli epicentri dei terremoti riportati in tabella 1.

Representation of earthquakes' epicentre reported in table 1. 


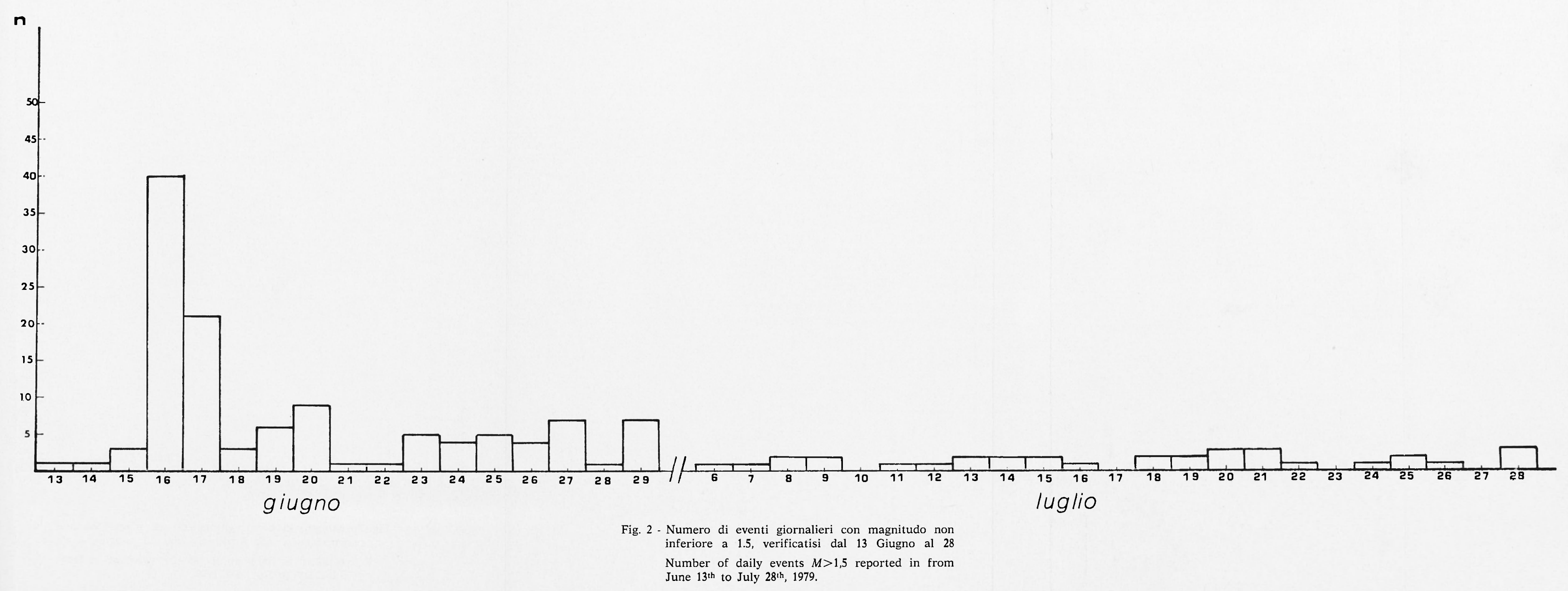




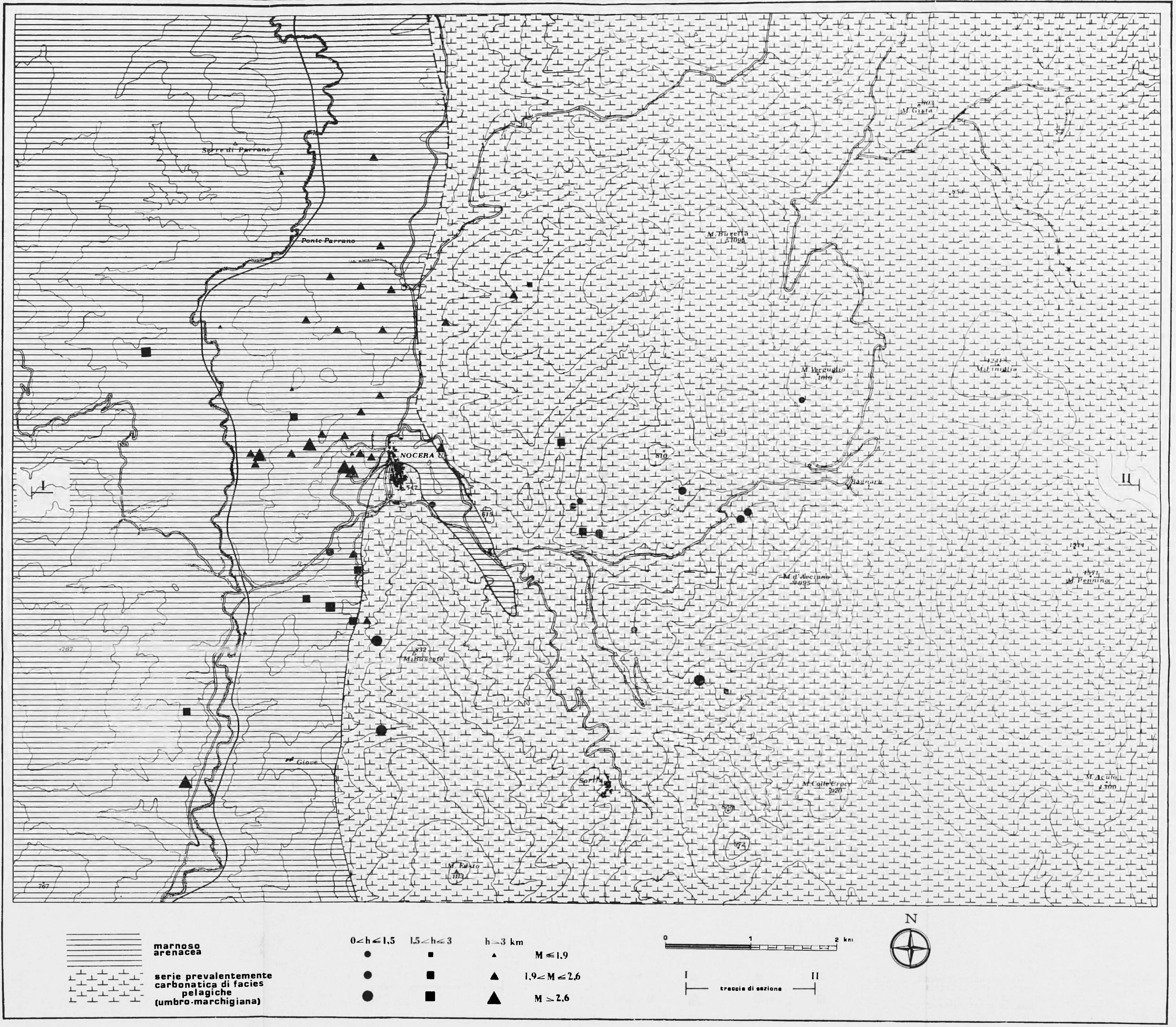

Fig. 3 - Rappresentazione degli epicentri dei terremoti riRepresentation of reported in earthquakes' epi- 


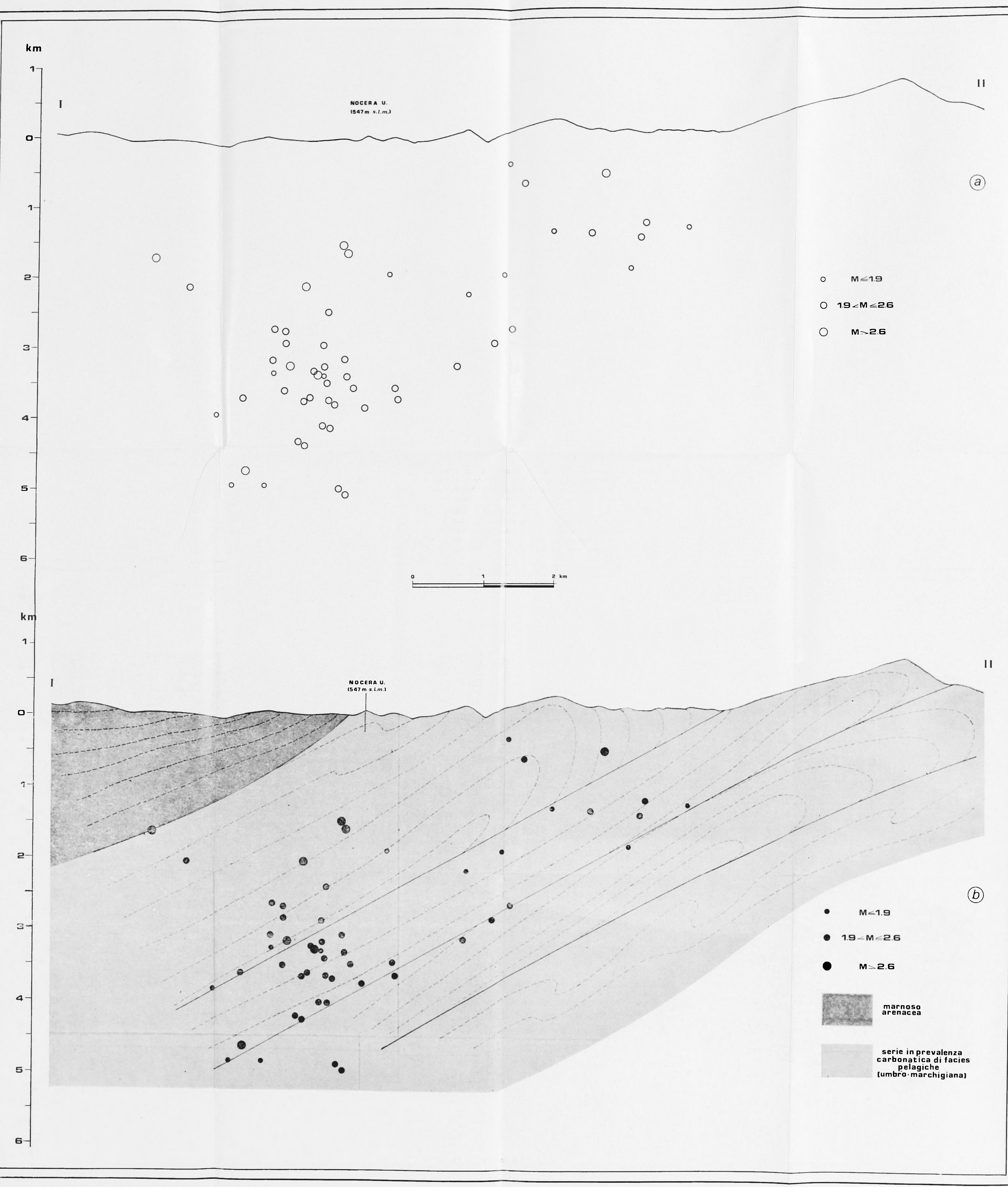

Fig. 4 - a) Distribuzione degli ipocentri dei terremoti ri-
portati in tabella 2 lungo un profilo Est-Ovest. portati in tabella 2 lungo un profilo Est-Ovest.
b) Distribuzione precedente sovrapposta all'andamento degli elementi strutturali affioranti, estramento degli elementi
polato verso il basso.

a) Distribution of earthquakes' hypocentres, in table 2, along an Est-West profile. b) Previous distribution laid upon the trend of outcropping
structural elements, with extrapolation downwards. 